\title{
Long-term Results of Slanted Recession of Bilateral Lateral Rectus Muscle for Intermittent Exotropia with Convergence Insufficiency
}

\author{
Ji Min Kwon, Soo Jung Lee \\ Department of Ophthalmology, Haeundae Paik Hospital, Inje University College of Medicine, Busan, Korea
}

Purpose: To evaluate the long-term efficacy of slanted lateral rectus recession in children for reducing distance and near exodeviation and near-distance deviation difference in intermittent exotropia with convergence insufficiency.

Methods: The medical records of 53 patients with convergence insufficiency intermittent exotropia who underwent slanted bilateral lateral rectus recession performed by a single surgeon and received follow-up for more than 12 months were retrospectively analyzed. Deviation angles at 1 day, 1 week, 1 month, 3 months, 6 months, and 12 months postoperatively and on the last visit were reviewed. Surgical success was defined as postoperative residual distance and near deviation angles $\leq 8$ prism diopters and a difference between the near and distance angles $\leq 8$ prism diopters.

Results: The mean duration of follow-up was 24 months (range, 12 to 61 months). On the last visit, the residual deviation angles were $\leq 8$ prism diopters in $75.5 \%$ for distance, $62.3 \%$ for near, and $81.1 \%$ for the near-distance difference. Surgical success was achieved in 31 (58.5\%) patients, and none of them manifested limitations in eye movements or diplopia at the last follow-up visit.

Conclusions: Slanted lateral rectus recession is an effective surgical method for reducing distance and near exodeviation and near-distance deviation difference in intermittent exotropia with convergence insufficiency.

Key Words: Intermittent exotropia, Near-distance difference, Ocular motility disorders, Slanted lateral rectus recession

Intermittent exotropia (IXT) has been classified into four types by Burian: basic, divergence excess, pseudo-divergence excess, and convergence insufficiency [1]. The convergence insufficiency type, characterized by near deviation exceeding the distance deviation by at least 10 prism diopters (PD), is uncommon in children with IXT and ac-

Received: March 11, 2019 Final revision: March 21, 2019

Accepted: June 4, 2019

Corresponding Author: Soo Jung Lee, MD, PhD. Department of Ophthalmology, Haeundae Paik Hospital, Inje University College of Medicine, 875 Haeundae-ro, Haeundae-gu, Busan 48108, Korea. Tel: 82-51-797-2310, Fax: 82-51-797-2321, E-mail: kris9352@paik.ac.kr counts for $1.2 \%$ to $7.8 \%$ [2].

Surgical treatments for this type of IXT include bilateral medial rectus (MR) resection with or without a slanting procedure, unilateral lateral rectus (LR) recession with MR resection, bilateral LR recession with or without a slanting procedure, and MR resection with an adjustable suture [3-8]. Since many surgical methods have been proposed, papers investigating and comparing the surgical effects have been reported [9-11]. Snir et al. [3] have reported a $92 \%$ success rate at one year after slanted LR recession for convergence insufficiency type IXT. Since only shortterm results of slanted LR recession of convergence insuf- 
ficiency IXT have been reported, this study was designed to evaluate the long-term efficacy of slanted LR recession on distance and near exodeviation and near-distance deviation difference in children with a near deviation angle exceeding the distance deviation angle by at least $10 \mathrm{PD}$.

\section{Materials and Methods}

This study retrospectively analyzed 53 patients with convergence insufficiency type exotropia who underwent slanted bilateral LR recession by a single surgeon (SJL) between February 2009 and September 2017 and were followed up for more than 12 months. Patients with a near deviation angle exceeding the distance deviation angle by at least 10 PD were defined as having convergence insufficiency type of exotropia.

Patients with other types of strabismus including paralytic or restrictive strabismus, amblyopia, other ocular diseases, history of ophthalmic surgery including prior strabismus surgery, nystagmus, congenital anomalies, neurologic abnormalities, and chromosomal or systemic diseases were excluded.

Preoperative best-corrected visual acuity and refractive errors were measured, and glasses were used when necessary. Following the cover-uncover test, an alternate prism cover test was performed at near $(33 \mathrm{~cm})$ and distance $(6$ $\mathrm{m})$. Preoperative stereopsis was evaluated by Titmus stereoacuity test (Stereo Optical, Chicago, IL, USA), and stereopsis worse than $100 \mathrm{arc} / \mathrm{sec}$ was defined as poor.

All the operations were performed under general anesthesia by the same surgeon. Following a forniceal-conjunctival incision, slanted LR recession was performed. The amount of recession of the upper pole was based on the distance deviation angle, and that of the lower pole was based on the near deviation angle. Surgical dose was based on Park's method. To calculate the corrective effect of slanted LR recession on the near-distance difference, the mean reduction in near-distance difference was divided by the mean difference between recession amounts of the upper and lower poles.

Deviation angles and limitation of eye movements were examined at 1 day, 1 week, 1 month, 3 months, 6 months, and 12 months postoperatively and on the last visit. The angle of deviation was considered positive for exodeviation and negative for esodeviation.
Surgical success was defined as postoperative residual distance and near deviation angles $\leq 8 \mathrm{PD}$ and a difference between near and distance angles $\leq 8$ PD [3]. Overcorrection, or consecutive esotropia, was defined as postoperative esodeviation $>8 \mathrm{PD}$, and recurrence was defined as postoperative exodeviation $>8 \mathrm{PD}$. The patients were divided into three groups according to deviation angle on the first day after the operation: esodeviation $>8 \mathrm{PD}$, residual deviation angle $\leq 8 \mathrm{PD}$, and exodeviation $>8 \mathrm{PD}$. The patients were classified as esodeviation or exodeviation if either the distance or near deviation angle exceeded 8PD. The difference in success rate was compared between the groups.

Statistical analyses were performed with the paired $t$-test and chi-square test using PASW Statistics ver. 18.0 (SPSS Inc., Chicago, IL, USA). A $p$-value less than 0.05 was considered statistically significant.

The study protocol was approved by the institutional review board of Inje University Haeundae Paik Hospital (HPIRB 2018-06-014-002) and adhered to the declaration of Helsinki. Informed consent was waived due to the retrospective nature of the study.

\section{Results}

A total of 60 patients with convergence insufficiency type exotropia who underwent slanted bilateral LR recession within the period between February 2009 and September 2017 were identified. Seven patients were excluded with insufficient follow-up. The inclusion criteria were met by 53 patients ( 23 boys and 30 girls). The mean age at the time of surgery was 7.5 years (range, 3 to 15 years), and mean duration of follow-up was 24 months (range, 12 to 61 months) (Table 1). The follow-up period was one year

Table 1. Preoperative patient characteristics

\begin{tabular}{lc}
\hline Characteristics & Value \\
\hline No. of patients & 53 \\
Sex & $23(43)$ \\
Male & $30(57)$ \\
Female & $7.5 \pm 2.2(3-15$, median 7$)$ \\
Age (yr) & $24 \pm 14.0(12-61$, median 17$)$ \\
Follow-up period (mon)
\end{tabular}

Values are presented as number (\%) or mean \pm standard deviation (range) unless otherwise indicated. 


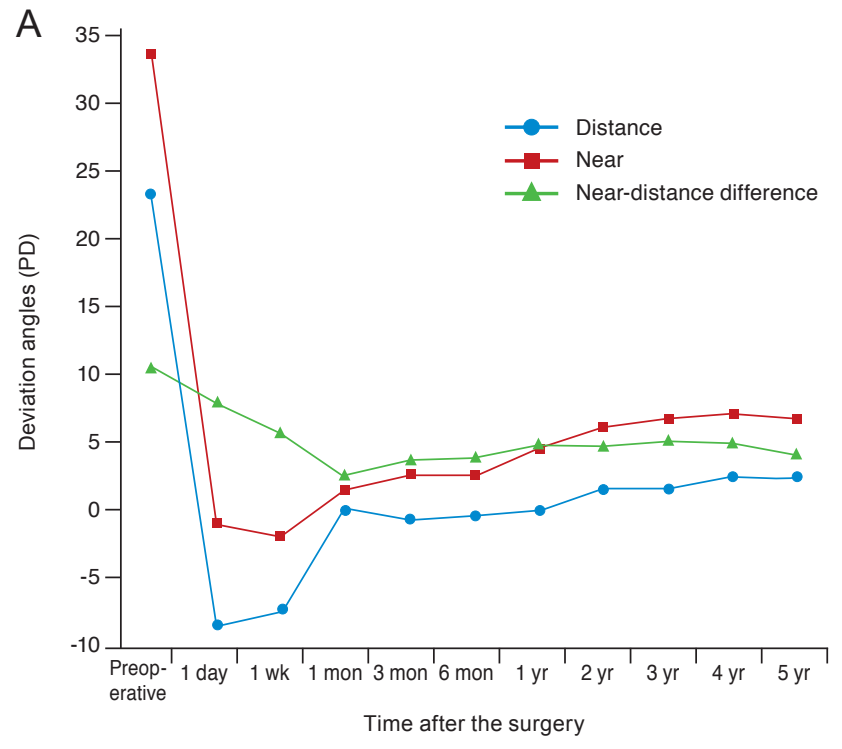

Fig. 1. Distance deviation, near deviation, and near-distance difference over time. The mean distance deviation angle decreased from 23.2 prism diopters (PD) preoperatively to 2.6 PD postoperatively, the mean near deviation angle decreased from $33.8 \mathrm{PD}$ preoperatively to $6.2 \mathrm{PD}$ postoperatively, and the mean difference between the near and distance angles decreased from 10.6 PD preoperatively to $4.0 \mathrm{PD}$ postoperatively.

in 30 patients, two years in nine patients, three years in nine patients, four years in four patients, and five years in one patient.

On the Titmus stereoacuity test performed before surgery, 47 patients showed stereopsis $\leq 100 \mathrm{arc} / \mathrm{sec}$, whereas six showed poor stereopsis $>100 \mathrm{arc} / \mathrm{sec}$. Mean preoperative stereopsis was $66.7 \mathrm{arc} / \mathrm{sec}$, and postoperative stereopsis was $65.1 \mathrm{arc} / \mathrm{sec}$, and there was no significant difference $(p>0.05)$. The mean surgical amount of recession was 5.6 $\mathrm{mm}$ (range, 4 to $8 \mathrm{~mm}$ ) in the upper pole and $7.3 \mathrm{~mm}$ (range, 6 to $9 \mathrm{~mm}$ ) in the lower pole. The mean difference between the recession amounts of the upper and lower poles was $1.7 \mathrm{~mm}$ (range, 1 to $3 \mathrm{~mm}$ ).

The mean distance deviation angle significantly decreased from $23.2 \pm 5.1 \mathrm{PD}$ (range, 14 to $40 \mathrm{PD}$ ) preoperatively to $2.6 \pm 7.6 \mathrm{PD}$ (range, -18 to $20 \mathrm{PD}$ ) postoperatively $(p<0.0001)$. The mean near deviation angle decreased significantly from $33.8 \pm 5.1 \mathrm{PD}$ (range, 25 to $50 \mathrm{PD}$ ) preoperatively to $6.2 \pm 10.2 \mathrm{PD}$ (range, -18 to $30 \mathrm{PD}$ ) postoperatively $(p<0.0001)$. The mean difference between the near and distance angles decreased significantly from 10.6 $\pm 1.5 \mathrm{PD}$ (range, 10 to $17 \mathrm{PD}$ ) preoperatively to $4.0 \pm 4.4$ $\mathrm{PD}$ (range, 0 to $16 \mathrm{PD})$ postoperatively $(p<0.0001)$ (Fig. 1$)$. Every millimeter of difference between the upper and lower poles of the slanted recession reduced the difference between the near and distance angles of exodeviation by 4.6 PD.

During follow-up, five patients showed overcorrection at distance, while 10 showed overcorrection at both near and distance. All patients with overcorrection were treated with alternating eye patching, and five of them showed improvement. Among the 10 patients who did not improve, six were prescribed prism glasses, and four underwent reoperation for consecutive esotropia. One of the six patients prescribed with prism glasses showed improvement and stopped using glasses, but five of them continued wearing them. None of the patients manifested limitation of eye movements or diplopia during follow-up.

Postoperative residual deviation angle $\leq 8 \mathrm{PD}$ was noted in $40(75.5 \%)$ patients for distance and in $33(62.3 \%)$ for near deviation. A near-distance difference $\leq 8$ PD was measured in $43(81.1 \%)$ patients. Surgical success indicating satisfaction of all three conditions was achieved in 31 $(58.5 \%)$ patients.

Recurrent exotropia occurred in four patients, two of the basic type and two with the convergence insufficiency type, and all of them underwent reoperation. At the last follow up, none of the patients demonstrated $\mathrm{A}-\mathrm{V}$ pattern misalignment.

Surgical success was achieved in 22 of 31 patients with esodeviation $>8 \mathrm{PD}$, seven of 18 patients with residual deviation angles $\leq 8 \mathrm{PD}$, and two of four patients with exodeviation $>8$ PD on the first day after the operation. There was no statistically significant difference in success rate among the three groups ( $p=0.084$ ).

During the minimum observation period of one year, success rates of deviation at distance and near and near-distance difference were highest at one month (Table 2).

\section{Discussion}

Convergence insufficiency type IXT is one of the four types of IXT classified by Burian [1] and refers to IXT with near deviation exceeding the distance deviation by at least 10 PD. To date, various attempts have been made not only using MR, but also LR to correct the distance and near exodeviation and to reduce the difference between near and distance deviation. Haldi [12] proposed bilateral MR resection and reported that near exodeviation de- 
Table 2. Success rates according to postoperative period

\begin{tabular}{|c|c|c|c|c|c|c|}
\hline Time after surgery & 1 day & $1 \mathrm{wk}$ & $1 \mathrm{mon}$ & 3 mon & 6 mon & $1 \mathrm{yr}$ \\
\hline No. of patients ${ }^{*}$ & 53 & 53 & 53 & 53 & 53 & 53 \\
\hline Distance deviation $^{\dagger}$ & $22(41.5)$ & $30(56.6)$ & $47(88.7)$ & $41(77.4)$ & $43(81.1)$ & $40(75.5)$ \\
\hline Near deviation & $42(79.2)$ & $45(84.9)$ & $45(84.9)$ & $39(73.6)$ & $38(71.7)$ & $30(56.6)$ \\
\hline Near-distance difference ${ }^{\S}$ & $27(50.9)$ & $38(71.7)$ & $49(92.5)$ & $43(81.1)$ & $48(90.6)$ & $43(81.1)$ \\
\hline Success $^{\Pi}$ & $17(32.1)$ & $30(56.6)$ & $43(81.1)$ & $35(66.0)$ & $37(69.8)$ & $27(50.9)$ \\
\hline
\end{tabular}

Values are presented as number (\%).

${ }^{*}$ Cumulative number of patients in the follow-up period; ${ }^{\circ}$ Number of patients with distance deviation angle $\leq 8$ prism diopters; ${ }^{t}$ Number of patients with near deviation angle $\leq 8$ prism diopters; ${ }^{8}$ Number of patients with a difference between near and distance deviation angles $\leq 8$ prism diopters; ${ }^{\Pi}$ Number of patients with distance and near deviation angles $\leq 8$ prism diopters and a difference between near and distance angles $\leq 8$ prism diopters).

creased to $<10 \mathrm{PD}$ in $50 \%$ of the patients. Kushner [13] also performed bilateral MR resection and reported an under-correction rate of $83 \%$ for the near deviation angle at one year postoperatively.

Based on the hypothesis that MR primarily affects near deviation and LR affects mainly distance deviation, unilateral MR resection and LR recession were performed [14]. Kraft et al. [15] reported that near deviation and near-distance difference decreased in all patients after unilateral augmented MR resection and LR recession; however, 18\% of the patients showed consecutive esotropia, and all the patients had abduction limitation.

Slanted LR recession was introduced by Snir et al. [3] based on Scott's investigation. Scott [14] reported that the fibers of the upper and lower poles of the LR muscle are the same length at the primary position, but the upper muscle fibers lengthen from 40.0 to $41.5 \mathrm{~mm}$ and the lower muscle fibers shorten from 40.0 to $37.1 \mathrm{~mm}$ during down gaze and in near vision. This means the lower fibers are shorter and thus stronger than the upper fibers at near vision, and by recessing or weakening the lower muscle fibers more than the upper fibers, exodeviation in near deviation can be reduced. Moreover, the near-distance difference can be reduced by balance of the upper and lower muscle fibers at the new insertion site.

Snir et al. [3] performed slanted LR recession in IXT patients with convergence insufficiency (unilateral surgery in seven patients and bilateral in five) and reported that residual distance and near exodeviation and near-distance difference were $\leq 8$ PD in $92 \%$ at 12 months postoperatively. Song and Lee [16] compared slanted bilateral LR recession and standard bilateral LR recession in convergence insuf- ficiency IXT and reported that the surgical success rate was significantly higher in the slanted group (35\% vs. $7 \%$ ). Chun and Kang [17] performed slanted bilateral LR recession and reported that residual distance and near exodeviation and near-distance difference were $\leq 8 \mathrm{PD}$ in $83.9 \%$ of patients at 6 months postoperatively.

In this study, the researchers performed slanted LR recession in 53 patients with IXT with convergence insufficiency. A 1-mm difference between the upper and lower poles of the slanted recession reduced the near-distance difference by 4.6 PD, which was consistent with the results of Snir et al. [3].

At the last follow-up, more than one year postoperatively, the residual deviation angles were $\leq 8 \mathrm{PD}$ in $75.5 \%$ for distance, $62.3 \%$ for near, and $81.1 \%$ for the near-distance difference. Out of 53 patients, 31 showed surgical success, which was defined as postoperative residual distance and near deviation angles $\leq 8 \mathrm{PD}$ and a difference between near and distance angles $\leq 8 \mathrm{PD}$. Consecutive esotropia was observed in nine patients $(16.9 \%)$.

Residual distance and near deviation angles and a difference between near and distance angles $\leq 8 \mathrm{PD}$ was obtained in $69.8 \%$ of patients at 6 months postoperatively and in $50.9 \%$ at 12 months postoperatively. These percentages were lower than those reported by Chun and Kang [17] and Snir et al. [3], which were $83.9 \%$ and $92 \%$, respectively; this could be due to the larger sample size in the current study compared to these previous two studies. Moreover, the mean age of patients in the current study was 7.5 years, while that of Chun and Kang [17] was 9.2 years, and that of Snir et al. [3] was 16.9 years. The possibility that a more accurate angle of deviation was not measured due to lack 
of cooperation might also attribute to the low success rate of the current study. Compared with other surgical methods, the surgical success rate at one year postoperatively was higher than that found with bilateral MR resection performed by Kushner [13], and the occurrence of consecutive esotropia was lower than that of unilateral MR resection and LR recession reported by Kraft et al. [15].

Kushner [18] reviewed insertion slanting recessions or biased resection for treating A- and V-pattern strabismus and asserted that it is the recession or resection itself, not the slanting, that affects the outcome. With convergence insufficiency IXT, however, higher success rates were reported in slanted recession compared to conventional LR recession $[16,19]$. Although the current study did not directly compare conventional and slanted recession for convergence insufficiency, it can be concluded that slanting surgery is effective for convergence insufficiency, as with previously reported results.

There have been reports that early overcorrections are related to final surgical success in exotropia [20-23]. In this study, there was no significant difference in final success rates between esodeviation $>8 \mathrm{PD}$, residual deviation angles $\leq 8 \mathrm{PD}$, and exodeviation $>8 \mathrm{PD}$ at 1 day postoperatively.

With respect to success rates during follow-up, distance and near deviation and near-distance difference were the highest at 1 month postoperatively. In distance deviation, success rates at 1 day and 1 week postoperatively were $41.5 \%$ and $56.6 \%$, respectively, which may be due to overcorrection observed in $58.5 \%$ and $43.4 \%$ of patients. The best success rate 1 month postoperatively in our study was attributed to high rates of overcorrection at distance 1 day and 1 week postoperatively.

The present study is limited by a relatively small population size, its retrospective nature, and lack of a control group. Additionally, the numbers of patients in the groups over the follow-up period were not even, resulting in inaccuracy of the comparison of success rates during follow-up. However, the research is meaningful as a study of relatively long-term postoperative results, with a mean follow-up period of 24 months.

In this study, slanted LR recession was found to be an effective technique for reducing not only distant and near deviation angles, but also the near-distance difference. In addition, it is considered a safe procedure with few complications such as abduction disorder and diplopia. More- over, the effect of slanted LR recession was maintained for more than one year in the current study, indicating it as an effective treatment for convergence insufficiency type IXT.

\section{Conflict of Interest}

No potential conflict of interest relevant to this article was reported.

\section{Acknowledgements}

This work was supported by the 2018 Inje University research grant.

\section{References}

1. Burian HM. Exodeviations: their classification, diagnosis and treatment. Am J Ophthalmol 1966;62:1161-6.

2. Kushner BJ, Morton GV. Distance/near differences in intermittent exotropia. Arch Ophthalmol 1998;116:478-86.

3. Snir M, Axer-Siegel R, Shalev B, et al. Slanted lateral rectus recession for exotropia with convergence weakness. Ophthalmology 1999;106:992-6.

4. Raab EL, Parks MM. Recession of the lateral recti: effect of preoperative fusion and distance-near relationship. Arch Ophthalmol 1975;93:584-6.

5. von Noorden GK. Resection of both medial rectus muscles in organic convergence insufficiency. Am J Ophthalmol 1976;81:223-6.

6. Choi MY, Hwang JM. The long-term result of slanted medial rectus resection in exotropia of the convergence insufficiency type. Eye (Lond) 2006;20:1279-83.

7. Choi MY, Hyung SM, Hwang JM. Unilateral recession-resection in children with exotropia of the convergence insufficiency type. Eye (Lond) 2007;21:344-7.

8. Choi DG, Rosenbaum AL. Medial rectus resection(s) with adjustable suture for intermittent exotropia of the convergence insufficiency type. J AAPOS 2001;5:13-7.

9. Wang B, Wang L, Wang Q, Ren M. Comparison of different surgery procedures for convergence insufficiency-type intermittent exotropia in children. $\mathrm{Br} J$ Ophthalmol 2014;98:1409-13. 
10. Ma L, Yang L, Li N. Bilateral lateral rectus muscle recession for the convergence insufficiency type of intermittent exotropia. J AAPOS 2016;20:194-6.

11. Farid MF, Abdelbaset EA. Surgical outcomes of three different surgical techniques for treatment of convergence insufficiency intermittent exotropia. Eye (Lond) 2018;32:693700.

12. Haldi BA. Surgical management of convergence insufficiency. Am Orthopt J 1978;28:106-9.

13. Kushner BJ. Exotropic deviations: a functional classification and approach to treatment. Am Orthopt $J$ 1988;38:8193.

14. Scott AB. Strabismus muscle forces and innervation. In: Lenerstrand G, Bach-y-Rita P, editors. Basic mechanism of ocular motility and their clinical implications: proceedings of the International Symposium Held in Wenner-Gren Center, Stockholm, June 4-6, 1974. New York: Pergamon Press; 1975. p. 181-91.

15. Kraft SP, Levin AV, Enzenauer RW. Unilateral surgery for exotropia with convergence weakness. J Pediatr Ophthalmol Strabismus 1995;32:183-7.

16. Song IJ, Lee SG. The effect of bilateral slanted lateral rec- tus recession in exotropia with near-far disparity. J Korean Ophthalmol Soc 2012;53:311-5.

17. Chun BY, Kang KM. Early results of slanted recession of the lateral rectus muscle for intermittent exotropia with convergence insufficiency. J Ophthalmol 2015;2015:380467.

18. Kushner BJ. Insertion slanting strabismus surgical procedures. Arch Ophthalmol 2011;129:1620-5.

19. Park JS, Lee SJ, Roh YB, Choi HY. Monocular slanted lateral rectus recession for exotropia with convergence insufficiency. J Korean Ophthalmol Soc 2007;48:1112-8.

20. Ruttum MS. Initial versus subsequent postoperative motor alignment in intermittent exotropia. J AAPOS 1997;1:88-91.

21. Raab EL, Parks MM. Recession of the lateral recti. Early and late postoperative alignments. Arch Ophthalmol 1969;82:203-8.

22. Cho YA, Kim SH. Postoperative minimal overcorrection in the surgical management of intermittent exotropia. $\mathrm{Br} J$ Ophthalmol 2013;97:866-9.

23. Ahn JH, Paik H. Long-term surgical outcomes of initial postoperative overcorrection in adults with intermittent exotropia. Korean J Ophthalmol 2018;32:228-33. 